\title{
Experiences of Using Wearable Computers for Ambient Telepresence and Remote Interaction
}

\author{
Mikael Drugge, Marcus Nilsson, Roland Parviainen, Peter Parnes \\ Luleå University of Technology \\ Department of Computer Science \& Electrical Engineering \\ Division of Media Technology \\ SE-971 87 Luleå, Sweden \\ \{mikael.drugge, marcus.nilsson, roland.parviainen, peter.parnes\}@csee.ltu.se
}

\begin{abstract}
We present our experiences of using wearable computers for providing an ambient form of telepresence to members of an e-meeting. Using a continuously running e-meeting session as a testbed for formal and informal studies and observations, this form of telepresence can be investigated from the perspective of remote and local participants alike. Based on actual experiences in real-life scenarios, we point out the key issues that prohibit the remote interaction from being entirely seamless, and follow up with suggestions on how those problems can be resolved or alleviated. Furthermore, we evaluate our system with respect to overall usability and the different means for an end-user to experience the remote world.
\end{abstract}

\section{Categories and Subject Descriptors}

H.4.3 [Information Systems Applications]: Communications Applications-Computer conferencing, teleconferencing and videoconferencing; H.5.1 [Information Interfaces and Presentation]: Multimedia Information Systems; H.5.2 [Information Interfaces and Presentation]: User Interfaces; H.5.3 [Information Interfaces and Presentation]: Group and Organization Interfaces

\section{General Terms}

Experimentation, Human Factors, Design.

\section{Keywords}

Ambient telepresence, mobile e-meetings, remote interaction, wearable computing.

\section{INTRODUCTION}

Wearable computing offers a novel platform for telepresence in general, capable of providing a highly immersive and subjective experience of remote events. By use of video, audio and personal annotations and observations, the user of a wearable computer can convey a feeling of "being there" even to those people who are not.

Permission to make digital or hard copies of all or part of this work for personal or classroom use is granted without fee provided that copies are not made or distributed for profit or commercial advantage and that copies bear this notice and the full citation on the first page. To copy otherwise, to republish, to post on servers or to redistribute to lists, requires prior specific permission and/or a fee.

ETP'04, October 15, 2004, New York, New York, USA

Copyright 2004 ACM 1-58113-933-0/04/0010 ...\$5.00.
The platform also enables a level of interaction between remote and local participants, allowing information to flow back and forth passing through the wearable computer user acting as a mediator. All in all, wearable computers emerge as a promising platform for providing telepresence, yet this statement also brings forward the following research questions:

- What form of telepresence can be provided using today's wearable computing technology?

- How can the telepresence provided be seamlessly used and employed in real-life scenarios?

- What is required to further improve the experience and simplify its deployment in everyday life?

In the Media Technology research group, collaborative work applications are used on a daily basis, providing each group member with an e-meeting facility from their regular desktop computer. In addition to holding more formal e-meetings as a complement to physical meetings, the applications also provide group members with a sense of presence of each other throughout the day. This latter case is referred to as the "e-corridor" - a virtual office landscape in which group members can interact, communicate and keep in touch with each other. As the e-corridor allows fellow coworkers to be together regardless of their physical whereabouts, it has become a natural and integrated part of our work environment.

As part of our ongoing research in wearable computing, we have had the wearable computer user join the e-corridor whenever possible; for example at research exhibitions, marketing events and student recruitment fairs. Since the members of our research group are already used to interact with each other through their desktop computers, we can build on our existing knowledge about e-meetings to study the interaction that takes place with a wearable computer user. This gives us a rather unique opportunity for studying the real-life situations that such a user is exposed to, and derive the strengths and weaknesses with this form of telepresence.

The key contribution of this paper is our experiences and observations of the current problems with remote interaction through wearable computing, and what obstacles must be overcome to make it more seamless. Furthermore, we propose solutions for how these shortcomings can be alleviated or resolved, and how that in turn opens up for further research in this area.

The organization of the paper is as follows: In section 2 we give a thorough introduction of our use of the e-corridor, serving as the basis for many of our observations and experiments. This is followed by section 3 in which we introduce our wearable computing research, and discuss how a wearable computer user can partake in 
the e-corridor. Section 4 continues by presenting our experiences of this form of telepresence, focusing on the shortcomings of the interaction, both from a technical as well as a social standpoint. The issues identified are subsequently addressed, followed by an overall evaluation of the system in section 5. Finally, section 6 concludes the paper together with a discussion of future work.

\subsection{Related Work}

Telepresence using wearable computers has been studied in a number of different settings. Early work by Steve Mann, et al., have explored using wearable computers for personal imaging [11, 13], as well as composing images by the natural process of looking around [12]. Mann has also extensively used the "Wearable Wireless Webcam" - a wearable computing equipment for publishing images onto the Internet, allowing people to see his current view as captured by the camera. Our work is similar to this in that we use wearable computers to provide telepresence, yet it differentiates itself by instead conveying the experience into an e-meeting session.

In computer supported cooperative work (CSCW), telepresence by wearable computers has often been used to aid service technicians in a certain task. Examples of this include [23] by Siegel et al. who present an empirical study of aircraft maintenance workers. This paper addresses telepresence that is not as goal-oriented as typical CSCW applications - instead, emphasis is laid on what ways there are to convey the everyday presence of each other, without any specific tasks or goals in mind. Roussel's work on the Well[22] is a good example of the kind of informal, everyday, communication our research enables.

A related example is the research done by Ganapathy et al. on tele-collaboration [6] in both the real and virtual world. This has similarities to our work, yet differs in that we attempt to diminish the importance of the virtual world, focusing more on bringing the audience to the real world conveyed by a remote user. The audience should experience a feeling of "being there", while the remote user should similarly have a feeling of them "being with him" — but not necessarily becoming immersed in their worlds.

In [8], Goldberg et al. present the "Tele-Actor" which can be either a robot or a wearable computer equipped human person at some remote location, allowing the audience to vote on where it should go and what it should do. A more thorough description of the "Tele-Actor" and the voting mechanism in particular can be found in [7]. The function of the "Tele-Actor" is similar to what is enabled by our wearable computing prototypes, but our paper focuses on providing that control through natural human to human interaction, rather than employing a voting mechanism.

As a contrast to using a human actor, an advanced surrogate robot for telepresence is presented by Jouppi in [9]. The robot is meant to provide a user with a sense of being at a remote business meeting, as well as give the audience there the feeling of having that person visiting them. The surrogate robot offers a highly immersive experience for the person in control, with advanced abilities to provide high quality video via HDTV or projectors, as well as accurately recreating the remote sound field. Besides our use of a human being rather than a robot, and not focusing on business meetings in particular, we investigate this area from the opposite standpoint: Given today's technology with e-meeting from the user's desktop, what kind of telepresence experience can be offered by a human user, and is that experience "good enough"?

In [1], a spatial conferencing space is presented where the user is immersed in the wearable computing world communicating with

\footnotetext{
${ }^{1}$ http://wearcam.org/
}

other participants. Another, highly immersive experience, is presented in [24] where Tang et al. demonstrate a way for two users to share and exchange viewpoints generated and explored using head motions. In contrast, our paper does not strive to immerse the user in the wearable computer, but rather provide the experience of an ambient, non-intrusive, presence of the participants. The motivation for this choice is that we want the participants to experience telepresence, and for that reason the remote user is required to remain focused on the real world - not immersed in a virtual world.

In [10], Lyons and Starner investigate the interaction between the user, his wearable computer and the external context as perceived by the user, for the purpose of performing usability studies more easily. Our paper reaches similar conclusions in how such a system should be built, but differentiates itself through our focus on telepresence rather than usability studies.

In [15], we present our experiences from sharing experience and knowledge through the use of wearable computers. We call this the Knowledgeable User concept, focusing on how information, knowledge and advice can be conveyed from the participants to the user of a wearable computer. In this paper, we instead discuss how this information can be conveyed in the other direction - from the remote side and back to a group of participants. Furthermore, we elaborate on this concept by discussing the current problems in this setup, our solutions to these, and how the end result allows us to achieve a more streamlined experience.

\section{EVERYDAY TELEPRESENCE}

In the Media Technology research group, collaborative work applications are used on a daily basis. Not only are regular e-meetings held from the user's desktop as a complement to physical meetings, but the applications run 24 hours a day in order to provide the group members with a continuous sense of presence of each other at all times. In this section, we will discuss how we use this so called "e-corridor" to provide everyday telepresence.

The collaborative work application that we use for the e-corridor is called Marratech Pro, a commercial product from Marratech $\mathrm{AB}^{2}$ based on earlier research [16] in our research group. Marratech Pro runs on an ordinary desktop computer and allows all the traditional ways of multimodal communication through use of video, audio and text. In addition, it provides a shared web browser and a whiteboard serving as a shared workspace, as well as application sharing between participants. Figure 1 shows the e-corridor as a typical example of a Marratech Pro session.

The members of our research group join a dedicated meeting session, the e-corridor, leaving the Marratech Pro client running in the background throughout the day. By allowing those in the group to see and interact with each other, this provides the members with a sense of presence of each other. Normally, each member works from their regular office at the university, using the client for general discussions and questions that may arise. Even though most members have their offices in the same physical corridor, the client is often preferred as it is less intrusive than a physical meeting. For example, for a general question a person might get responses from multiple members, rather than just a single answer which a physical visit at someone's office may have yielded. Similarly, each member can decide whether to partake in a discussion or not, based on available time and how much they have to contribute. The ambient presence provided by running the client throughout the day allows members to assess their fellows' workload, glance who are present or not, and in general provide a feeling of being together as a group.

However, providing presence for people who are still physically

\footnotetext{
$\overline{{ }^{2} \text { http://www.marratech.com/ }}$
} 


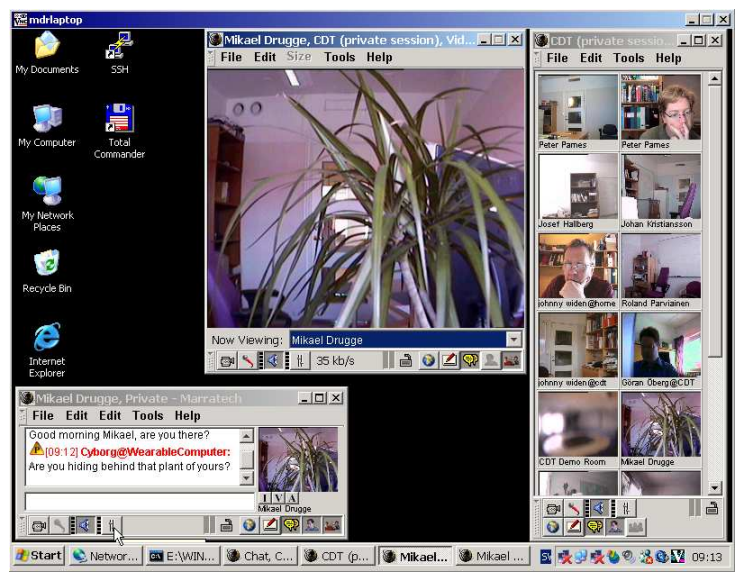

Figure 1: A snapshot of a typical Marratech Pro session.

close to each other is not everything; the true advantage of using the e-corridor becomes more apparent when group members are situated at remote locations. The following examples illustrate how the e-corridor has been used to provide a sense of telepresence for its members.

Working from home. Sometimes, a person needs to work from their home for some reason; maybe their child has caught a cold, or the weather is too bad to warrant a long commuting distance. In such situations, rather than becoming isolated and only use phone or email to keep in touch with the outside world, the e-corridor is used to get a sense of "being at work" together with their fellow co-workers.

Living in other places. In our research group, some members have for a period of time been living in another city or country, and thus been unable to commute to their regular office on a daily, weekly or even monthly basis. For example, one doctorand worked as an exchange student in another country for several months, while another person for over a year lived in a city hundreds of miles away. By using the e-corridor, the feeling of separation became significantly diminished; as testimonied by both the remote person as well as the local members remaining, it was sometimes difficult to realize that they were physically separated at all.

Attending conferences. As members of the research group travel to national or international conferences, they have been accustomed to enjoy their fellow co-workers' company regardless of time or place. For example, during long and tedious hours of waiting in the airport, members often join the ecorridor to perform some work, discuss some issue, or simply to chat with people in general. When attending the conference, the remote member can transmit speeches with live video and audio to the e-corridor, allowing people who are interested in the topic to listen, follow the discussion, and even ask questions themselves through that person. If the remote person is holding a presentation, it has often been the case that the entire research group has been able to follow it; encouraging, listening to, and providing support, comments and feedback to the presenter. In a sense, this allows the entire research group to "be there" at the conference itself, and it also allows the remote person to experience a similar feeling of having the group with him.
The seemingly trivial level of presence provided in ways like those described above should not be underestimated; even with simple means, this form of ambient everyday telepresence can have a strong influence on people and their work. Another testimony of the importance of this form of subtle, ambient, presence can be found e.g. in [19], where Paulos mentions similar awareness techniques for attaining user satisfaction.

Subsequently, by enabling a wearable computer user to join the e-corridor, the participants should be able to experience an encompassing form of telepresence. The remote user should similarly be able to feel the participants as "being with him", but not necessarily becoming immersed in the same way as they are.

\section{WEARABLE COMPUTERS}

In this section our wearable computer prototypes are presented, focusing on the hardware and software used to allow the prototypes to function as a platform for telepresence.

In terms of hardware, the wearable computer prototypes we build are based entirely on standard consumer components which can be assembled. The reason for favouring this approach, rather than building customized or specialized hardware, is that it allows for easy replication of the prototypes. For example, other researchers or associated companies who wish to deploy a wearable computing solution of their own, can easily build a similar platform.

The current prototype consists of a backpack containing a Dell Latitude C400 laptop with built-in IEEE 802.11b wireless network support. The laptop is connected to an M2 Personal Viewer headmounted display, with a web camera mounted on one side providing a view of what the user sees. Interaction with the computer is done through a Twiddler2 hand-held keyboard and mouse, and a headset is provided for audio communication. Figure 2 shows the prototype when being worn by one of the authors. This setup allows the user of the wearable computer to interface with a regular Windows XP desktop, permitting easy deployment, testing and studying of applications for mobility.

To perform studies on remote interaction and telepresence, the platform needs suitable software - in our case, we have chosen to run the Marratech Pro client. Figure 3 shows the user's view of the application as seen through the head-mounted display.

There are both advantages and disadvantages with using an existing e-meeting application, such as Marratech Pro, for the prototype. The main advantage is that it provides a complete, fully working, product that our research group already uses on a daily basis. This is, naturally, a desirable trait rather than "reinventing the wheel" by developing an application for mobile communication from scratch. It should be noted that as the product is a spin-off from previous research, we have access to the source code and can make modifications if needed, adapting it gradually for use in wearable computing scenarios. The second, perhaps most important advantage, is that the client allows us to participate in the e-corridor. This makes studies, observations and experiments on wearable computing telepresence easy to deploy and setup.

The disadvantage that we have found lies in the user interface which, albeit suitable for ordinary desktop computing, can become very cumbersome to use in the context of wearable computing. This observation holds true for most traditional WIMP ${ }^{3}$ user interfaces, for that matter; as noted e.g. by Rhodes in [20] and Clark in [3], the common user interfaces employed for desktop computing become severely flawed for wearable computing purposes. Although the user interface is not streamlined for being used in wearable computing, it remains useable enough to allow a person to walk around

\footnotetext{
${ }^{3}$ Windows, Icons, Menus, Pointer.
} 


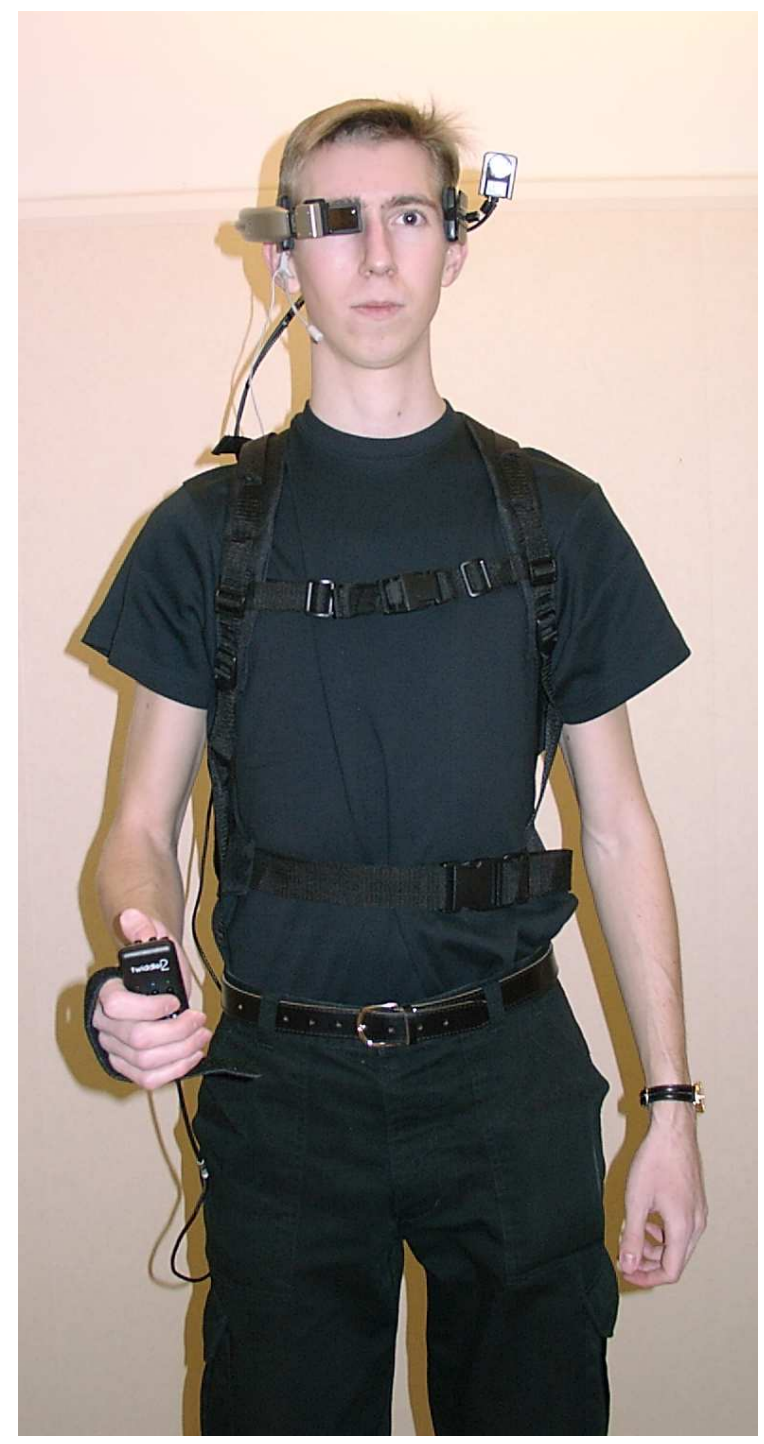

Figure 2: The wearable computer prototype being worn by one of the authors.

while taking part in e-meetings. Furthermore, the problems that emerge actually serve to point out which functions are required for wearable computing telepresence, allowing research effort to go into solving those exact issues. In this way, focus is not aimed at developing the perfect wearable user interface from scratch, as that can risk emphasizing functionality that will perhaps not be frequently used in the end. Rather, in taking a working desktop application, the most critical flaws can be addressed as they appear, all while having a fully functional e-meeting application during the entire research and development cycle.

\section{EXPERIENCES OF TELEPRESENCE}

In this section, the experiences of using a wearable computer for telepresence in the e-corridor will be discussed. The problems that arose during those experiences will be brought forward, together with proposals and evaluations on how those issues can be resolved.

The wearable computer prototype has mainly been tested at different fairs and events, providing a telepresence experience for people within our research group as well as to visitors and students.

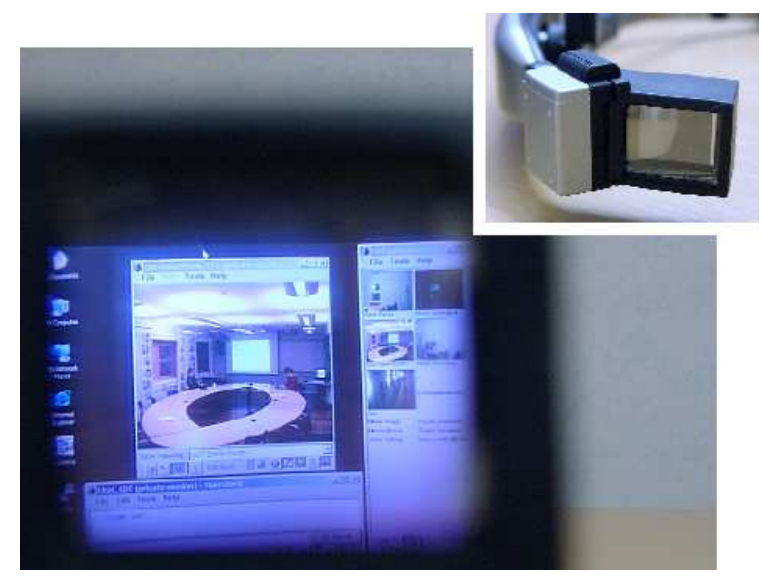

Figure 3: The Marratech Pro client as seen through the user's head-mounted display.

The fairs have ranged from small-scale student recruitment happenings, medium-sized demonstrations and presentations for researchers and visitors, and to large-scale research exhibitions for companies and funding partners. The prototype has been used in the local university campus area, as well as in more uncontrolled environments - e.g. in exhibition halls in other cities. In the former case, the necessary wireless network infrastructure have been under our direct control, allowing for a predictive level of service as the user roams the area covered by the network. However, in the latter case, the network behaviour is often more difficult to predict, occasionally restricting how and where the user can walk, and what quality of the network to expect. Both these cases, and especially the latter, serve as valuable examples on the shifting conditions that a wearable computer user will, eventually, be exposed to in a realworld setting. We believe it is hard or impossible to estimate many of these conditions in a lab environment, warranting this kind of studies being made in actual real-life settings.

When using a wearable computer for telepresence, unexpected problems frequently arise at the remote user's side - problems that are at times both counter-intuitive and hard to predict. These need to be resolved in order to provide a seamless experience to the audience, or else the feeling of "being there" risk being spoiled. Below follows the primary issues identified during the course of our studies.

\subsection{User Interface Problems}

As mentioned previously, the common WIMP user interfaces employed on the desktop does not work well in wearable computing. The primary reason for this is that the graphical user interface requires too much attention and too fine-grained level of control, thereby causing interference with the user's interaction with the real world. What may not be entirely apparent, however, is that these problems in turn can have severe social implications for the user, and those in turn interfere and interrupt the experience given to the audience.

As an example, the seemingly trivial task for the user to mute incoming audio will be given. This observation was initially made at a large, quite formal fair, arranged by funding partners and companies, but we have experienced it on other occasions as well. In order to mute audio, the collaborative work application offers a small button, easily accessible through the graphical user interface with a click of the mouse. Normally, the remote user received incoming audio in order to hear comments from the audience while walking 
around at the fair. However, upon being approached by another person, the user quickly wanted to mute this audio so as to be able to focus entirely on that person. It was at this point that several unforeseen difficulties arose.

The social conventions when meeting someone typically involves making eye-contact, shaking hands while presenting yourself, and memorizing the other person's name and affiliation. The deceptively simple task of muting incoming audio involves looking in the head-mounted display (preventing eye-contact), using the handheld mouse to move the pointer to the correct button (preventing you to shake hands), trying to mute the incoming audio (currently preventing you to hear what the other person says). These conflicts either made it necessary to ignore the person approaching you until you were ready, or to try to do it all at once which was bound to fail. The third alternative, physically removing the headset from the ear, was often the most pragmatical solution we chose to use in these situations.

Although this episode may sound somewhat humorous, which it in fact also was at that time, there are some serious conclusions that must be drawn from experiences like this. If such a simple task as muting audio can be so difficult, there must surely be a number of similar tasks, more or less complex, that can pose similar problems in this kind of setting. Something as trivial as carrying the Twiddler mouse and keyboard in the user's hand, can effectively prevent a person, or at least make it more inconvenient, to shake hands with someone. As the risk of breaking social conventions like this will affect the experience for everyone involved - the remote user, the person approaching, and the audience taking part — care must be taken to avoid this type of problems.

The specific situation above has been encountered in other, more general forms, on several occasions. The wearable computer allows for the remote user to work even while conveying live audio and video back to participants. An example of when this situation occurs is when the remote user attends a lecture. The topic may not be of immediate interest to the remote user, thereby allowing her to perform some other work with the wearable computer in the meantime. However, those persons on the other side who are following the lecture may find it interesting, perhaps interesting enough to ask a question through the remote user. In this case, that user may quickly need to bring up the e-meeting application, allowing her to serve as an efficient mediator between the lecturer and the other persons. In our experience, this context switch can be difficult with any kind of interface, as the work tasks need to be hidden and replaced with the e-meeting application in a ready state. The cost in time and effort in doing context switches like this effectively prevents a fully seamless remote interaction.

With the goal of providing a seamless and unhindered experience of telepresence, the user interface for the remote user clearly needs to be improved in general. Rather than trying to design the ideal user interface - a grand endeavour that falls outside the scope of this paper - we propose three, easy to implement, solutions to the type of problems related to the user interface of a wearable telepresence system.

- Utilize a "Wizard of Oz" approach [4]. It is not unreasonable to let a a team member help controlling the user interface of the remote user, especially not since there is already a group of people immersed in the remote world. We have done some preliminary experiments on using $\mathrm{VNC}[21]$, allowing a person sitting at his local desktop to assist the user of the wearable computer by having full control of her remote desktop. For example, typing in long URL:s can be difficult if one is not accustomed to typing on a Twiddler keyboard, but through VNC the assistant can type them on a keyboard on demand from the remote user. In a similar experiment, one person followed the remote user around, using a PDA with a VNC client running that allowed him to give assistance. It should be noted that this solution still offers some form of telepresence for the assistant, as that person can still see, via the remote desktop, a similar view as would have been seen otherwise.

- Automatically switch between real and virtual world. Even a trivial solution such as swapping between two different desktops - one suitable for the real world (i.e. the e-meeting application for telepresence), and the other suitable for work in the virtual domain (i.e. any other applications for work or leisure that the remote user may be running) — would make life simpler. By letting the switch be coupled to natural actions performed, e.g. sitting down, standing up, holding or releasing the Twiddler, the user is relieved of the burden of having to actively switch between two applications. The advantage may be small, but it can still be significant for efficiently moving between the real and virtual worlds.

- Reduce the need for having a user interface at all. ${ }^{4}$ Plain and simple, the less the remote user has to interact with the computer, the more he can focus on conveying the remote location to the audience. The hard part here is to find a proper balance, so that the remote user can still maintain the feeling of having his group present and following him.

\subsection{Choice of Media for Communicating}

For verbal communication, Marratech Pro offers both audio and text. Either media is important to have access to at certain occasions, as evidenced by our experiences described in [15]. As the wearable computer user is exposed to a number of different scenarios, being able to change between these media is a prerequisite for the communication to remain continuous and free from interruptions. For example, in the case discussed above, the remote participants' spoken comments interfered with the user's real world spoken dialogue. Rather than muting audio, a better solution would have been if the participants had instead switched over to sending their comments as text. This is something that is relatively simple to enforce by pure social protocols; as the participants are already immersed in the world that the user presents, they will be able to determine for themselves when it is appropriate to speak or not. However, although users can switch media at their own choice, this is not an ideal solution for seamless communication. For example, it requires participants to consciously care about which media to use, and does not take in account that they in turn may prefer one media over another for some reason.

To alleviate the problem of having all participants agree on using the same media, we have developed a prototype group communication system in Java that can arbitrarily convert between voice and text. Running the prototype, a user can choose to send using one media, while the receiver gets it converted to the other media. For example, a wearable computer user can choose to receive everything as text, while the other participants communicate by either spoken or written words. As speech recognition and voice synthesis techniques are well researched areas, the prototype is built using standard consumer products offering such functionality; currently the Microsoft Speech SDK $5.1^{5}$ is used.

\footnotetext{
${ }^{4}$ If a user interface is still required for some reason, our research in the Borderland architecture [14] intends to provide ubiquitous access to the tools needed.

${ }^{5} \mathrm{http}: / /$ www.microsoft.com/speech/
} 
The architecture for the system can be seen in figure 4 . The system accepts incoming streams of audio or text entering through the network, which are then optionally converted using speech recognition or voice synthesis, before they are presented to the user. Similarly, outgoing streams can be converted before they reach the network and are transmitted to the other participants. In practice, the implementation cannot perform speech recognition at the receiving side, nor voice synthesis at the sending side, due to limitations in the speech SDK currently used. Both of these conversions are, however, fully supported at the opposite sides.

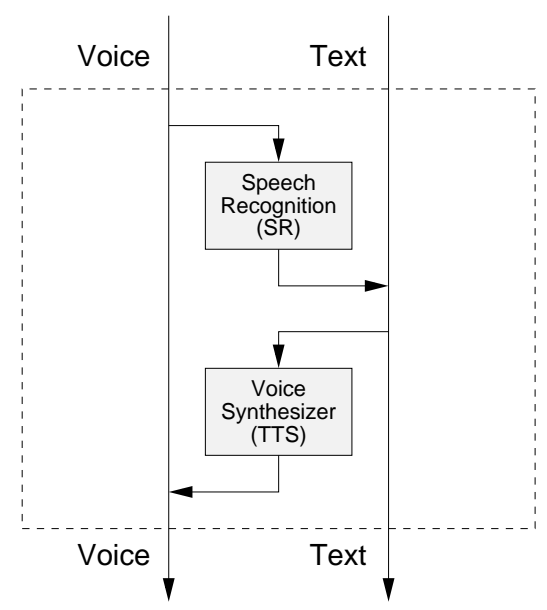

Figure 4: Architecture of the voice/text converter prototype, enabling communication across different media.

The prototype allows the choice of conversions being made to be controlled both locally and remotely. This means that participants can choose by what media communication from the remote user should be conveyed. For example, the remote user may lack any means for entering text, forcing her to rely solely on sending and receiving audio for communication. The participants, on the other hand, may prefer to communicate via text only. E.g. for a person attending a formal meeting, the only way to communicate with the outside world may be sending and receiving text through a laptop. The person in the meeting may therefore request the remote prototype to convert all outgoing communication to text. Similarly, the remote user has his prototype synthesizing incoming text into voice. In this way, a group of people can communicate with each other, with each person doing it through their preferred media.

The prototype runs under Windows XP serving as a proof of concept. Initial experiments have been performed using it for communication across different media. In the experiment, three persons held a discussion with each other, with each person using a certain media or changing between them arbitrarily. The results of these experiments indicate that this is a viable way of enabling seamless communication. Naturally, there are still flaws in the speech recognition, and background noise may cause interference with the speaker's voice. Nevertheless, as further progress is made in research on speech recognition, we consider a system like this will be able to provide a more streamlined experience of telepresence.

\section{EVALUATION}

In this section we will give an overall evaluation of our wearable system for telepresence. Emphasis will be placed on evaluating its overall usability and the different means for how an end-user can experience and interact with the remote world.

\subsection{Time for Setup and Use}

The time to setup the system for delivering an experience depends on how quickly participants and wearable computer users can get ready. The strength of our approach of utilizing Marratech Pro and the e-corridor, is that the software is used throughout the day by all participants. This means that in all experiments we have performed, we have never had any requirements for persons to e.g. move to a dedicated meeting room, start any specific application, or to dedicate a certain timeslot to follow the experience. For them, the telepresence becomes an ambient experience that can be enjoyed as much or as little as desired, all from the comfort of their own desktop.

As for the user equipped with a wearable computer, the setup time is often much longer due to the reasons listed below.

- The backpack, head-mounted display, headset and Twiddler are surprisingly cumbersome to put on and remove. Even though everything is relatively unobtrusive once fully worn, the time to actually prepare it is too long; for example, the head-mounted display needs to be arranged properly on the user's head, and cables become intertwined more often than not. All this makes the wearable computer less used in situations that warrant its use within short notice.

- The batteries for the laptop and the head-mounted display needs to be charged and ready for use. As this can not always be done with just a few hours worth of notice, this effectively prevents rapid deployment of the wearable computer to capture a certain event.

- The time for the laptop to start, together with gaining a network connection and launching the e-meeting application, is about 5 minutes in total - this is too long to be acceptable.

These are relatively minor problems, yet in resolving these the wearable computer can become more easily used for telepresence experiences than it is today. We consider this to be a prerequisite before it will be commonly accepted outside of the research area as a viable tool for telepresence. Therefore, in order to overcome these limitations, the next generation wearable system we design shall exhibit the properties listed below.

- By using a vest instead of a backpack containing the wearable computer, the head-mounted display, headset and Twiddler can be contained in pockets. This way, they remain hidden until the vest is fully worn and the user can produce them more easily.

- By using an ordinary coat hanger for the vest, a "docking station" can easily be constructed that allows battery connectors to be easily plugged in for recharging. This also makes using the vest-based wearable computer more natural, and thus also more easily used and accepted by the general public.

- By having the wearable computer always on or in a hibernated state when not worn and used, it allows easy restoration of the e-meeting so that anyone can wear and operate it within short notice.

These properties will serve to make the wearable computer easier to wear and use, thereby making it possible for anyone to wear it in order to deliver an experience of telepresence. 


\subsection{Different Levels of Immersion}

The e-corridor normally delivers a live stream of information (e.g. video, audio, chat, etc.) which the participants can choose to immerse themselves in. Typically, this is also the most common way of utilizing e-meeting applications like this. However, previous research in our group has added other ways of being part of an e-meeting; the first is a web interface [18], while the second is a history tool [17]. This gives us three distinct levels for how the telepresence can be experienced.

Marratech Pro. Using the e-meeting application, a live stream of video and audio allows the participants to get a first-hand experience of the event. The participants can deliver comments and instructions for the remote user, giving them a feeling of "being there" and allowing some degree of control of that user. Similarly, the remote user can deliver annotations and comments from the event, increasing the participants' experience further. What they say, do, and desire all have an immediate effect on the whole group, making the immersion very encompassing.

Web interface. Occasionally, persons are in locations where the network traffic to the e-meeting application is blocked by firewalls, or where the network is too weak to deliver live audio and video streams. To deal with such occasions, research was done on a web interface [18] that provides a snapshot of the current video, together with the full history of the textbased chat. The web interface can be seen as a screenshot in figure 5. Accessing this interface through the web, participants can get a sense for what is currently going on at the moment. Although they are not able to get a live, streaming, experience, the web interface has proven to work good enough to allow participants to control and follow the wearable computer user around.

For example, at one occasion, a person used to doing demonstrations of the wearable computer was attending an international conference, the same day as a large exhibition was to take place at his university back home. As he was away, another person had to take on his role of performing the demonstration. Due to problems in the network prohibiting the regular e-meeting client to run properly, the web interface was the only possible way of joining the e-corridor. Nevertheless, this allowed him to follow that remote user during the demonstration - offering advice and guidance, and even being able to talk (through the remote user) to persons he could identify in the video snapshots. For this person, the web interface allowed him to "be" at the demonstration, while he in fact was in another country, and another time zone for that matter, waiting for the conference presentations to commence. This example serves to illustrate that very small means seem to be necessary to perform effective telepresence, and also how a user can seamlessly switch between different levels of immersion and still have a fruitful experience.

History tool. The history tool [17] is a research prototype that captures and archives events from an e-meeting session. A screenshot of the tool can be found in figure 6. The tool allows people to search for comments or video events, as well as browse it in chronological order to basically see what has happened during the last hours, days or weeks (e.g to see whether a meeting has taken place or not). Snapshots of the video for a particular user are recorded whenever a user enters a chat message, together with the text message itself and

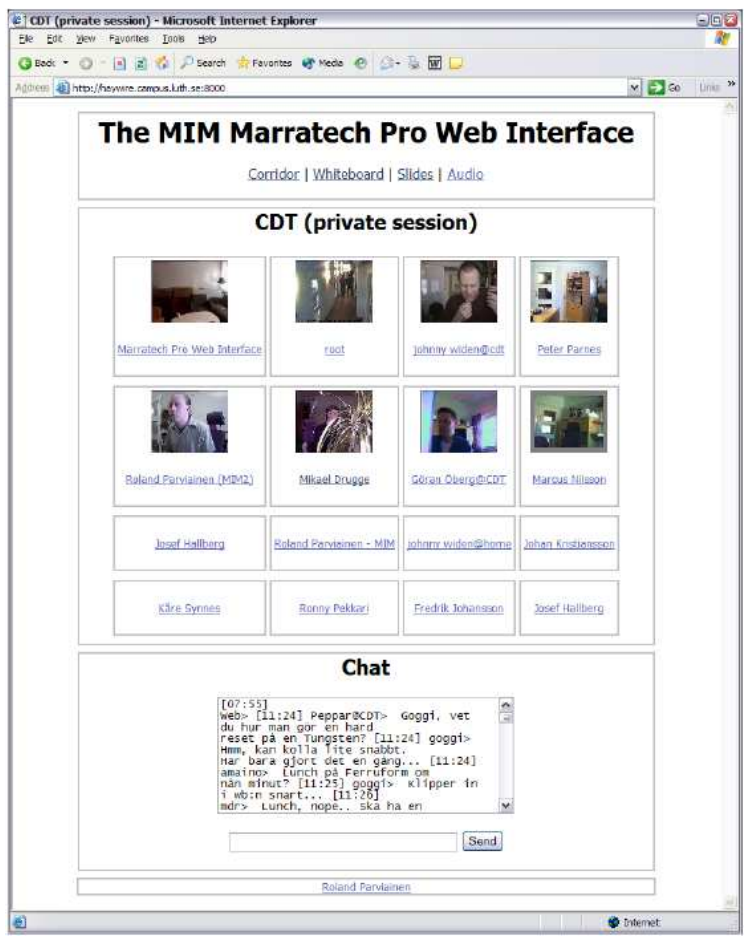

Figure 5: A screenshot of the Marratech Pro web interface, allowing access to e-meetings via web browsers.

the time when it was written. Using motion detection techniques, snapshots are also taken whenever something happens in the video stream. E.g. when a person enters or leaves their office, video frames from a few seconds before and after the triggering event will be recorded, thus being able to see whether that person is actually entering or leaving the room. Naturally, this is mainly suitable and used for clients equipped with a stationary camera, because a head-mounted camera tends to move around a lot causing most video to be recorded. Furthermore, events related to a single person can be filtered out in order to follow that particular person during the course of a day, for example.

In terms of telepresence, the tool is, as the name suggests, a history tool and as such does not offer any means for in-

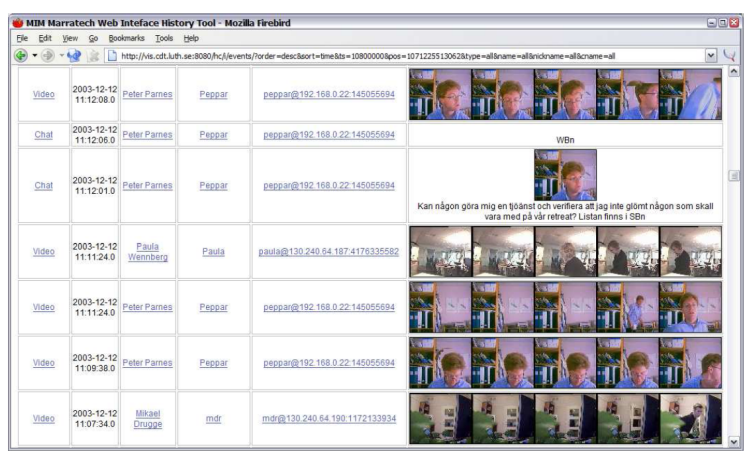

Figure 6: A screenshot of the Marratech Pro history tool, archiving events of interest. 
teracting with the persons ${ }^{6}$. However, it serves as a valuable starting point for someone who has missed the beginning of e.g. the coverage of a certain exhibition, and who wants a summary and recap of the course of events so far. This may be done in order to prepare the user for becoming more immersed when following the rest of the coverage live, something which can be more easily done having first received the summary information as a primer.

The advantage of using the history tool, rather than letting the user watch a complete recording of the events so far, is that the tool often tends to manage capturing the events that are of key interest. For example, as something is seen by or through the wearable computer user, the amount of chat and conversations often rise, thereby capturing a large amount of video as well as audio clips around that point in time. In this way, the history tool serves as an efficient summary mechanism that implicitly captures events of interest; the more interest, the more conversations and actions, and the more will be archived and subsequently reviewed. After having gone through the history tool, the user can easily switch to more live coverage via the client or web interface. Thus, the history tool serves to make the transgression from the real world to the immersion in the remote world more seamless.

\subsection{Appearance and Aesthetics}

We have found that the appearance and looks of the wearable computer can dramatically influence the audience's experience of telepresence. What we mean by this statement is that the user of a wearable computer tends to stand out among a crowd, often drawing a lot of attention causing more people to approach the person out of curiosity - more so than what would have been the case without wearing the computer. Sometimes, people even become intimidated by being confronted with a "living computer" — again, causing people to react in ways they would not normally do. Although the effects are not always negative ${ }^{7}$, it is important to be aware of the fact that they do exist and that they will, invariably, affect how the remote location is perceived. This becomes even more important to bear in mind considering that the audience may have no idea that this takes place, thereby being given a flawed or at least skewed perception of the remote location.

As telepresence should, in our opinion, be able to offer the participants a representation of a remote location that is as true and realistic as possible, measures need to be taken to ensure that the wearable computer will blend in with its user and the surrounding environment. For this reason, our next generation wearable computer will be smaller and designed to hide the technology as much as possible, according to the following criterias.

- A head-mounted display is difficult to hide and, due to its novelty, draws a lot of attention. With a smaller display, optionally mounted on a pair of glasses, it will be less noticed and easier to hide. At the same time, it becomes easier to motivate its use when people ask questions - motivating the use of a large, bulky display does not tend to sound credible to most people we have met. The less focus that is laid on the technology permitting telepresence, the more effective will it be.

- Eye-contact is very important; our experiences have shown that for efficient social interaction, both parties need to see

\footnotetext{
${ }^{6}$ For any interaction, either the Marratech Pro client or the web interface can be used.

${ }^{7}$ On the contrary, wearable computers often generate much attention and numerous socially beneficial interactions with people.
}

both of each others' eyes. A semi-transparent head-mounted display allows the remote user to get eye-contact, yet one eye remains obscured from the other person's viewpoint. In this respect, the choice of a semi-transparent or opaque display has little impact on telepresence - the primary requirement is that it allows for eye-contact so that the experience delivered is not hindered.

- The camera is very important as it conveys video to the other participants. As discussed in [5], there are benefits and drawbacks with different placements, so a definite answer is hard to give for the case of providing good telepresence. Also, from a socio-technical standpoint, the question is whether the camera should be hidden well enough not to disturb the scene it captures, or if it should remain visible to let people know their actions are being conveyed to other people watching. For the time being, the camera for our wearable computer will remain head-mounted and visible to the spectators, since this allows us to effectively convey the scene with a relatively modest level of disturbance.

Referring to the previous discussion regarding eye-contact; in terms of allowing the audience to "meet" with a remote person seen through the wearable computer, they must be given the impression of eye-contact with that person. In [2], Chen presents a study of how accurately persons can perceive eye-contact. The results can be interpreted as suggesting the upper part of the head, rather than the areas in the lower part or shoulder areas, as the proper position for a head-mounted camera. Such placement, e.g. on top of the user's head or at the sides (as it is in our current setup), should provide a feeling of eye-contact for the audience, without drawing too much attention from the user. However, a more formal user study is required to validate this hypothesis of proper placement for eye-contact with a wearable camera.

- The Twiddler mouse and keyboard is currently a prerequisite for interacting with the wearable computer, yet as discussed before in section 4.1, it also interferes with the user's interactions in the real world. However, for the sole purpose of providing telepresence, the only interaction that is actually required on behalf of the remote user is when comments need to be entered as text. This observation means that if the participants can cope without such feedback, it will free the remote user's hands and allow for a more effective interaction with the remote environment. This, in turn, should make for a better experience that is not interrupted by the technology behind it. Of course, there is still the question whether this benefit outweighs the lack of textual comments, but that is likely to vary depending on the event that is covered. There may also be other types of keyboard which are less likely to cause this kind of problems, although we have only utilized the Twiddler so far in our experiments.

- Using a vest rather than a backpack to hold the computing equipment will enable the user to move around, and especially sit down, much more comfortably. With a backpack, the user lacks support for his back when sitting or leaning against objects, at the same time the added weight of the batteries and laptop cause fatigue in the area of shoulders and neck. This fatigue tends to reduce the physical movement of the remote user after long hours of covering an event, and this is detrimental for the audience and serves to reduce their motivation for following the event. Also, to allow for an immersive telepresence, the remote user should be able to partake 
in social activities - especially something as simple such as sitting down discussing with someone over a cup of coffee. Using a vest, the weight and computing equipment is distributed over a larger part of the user's body, thereby making it less obtrusive and permitting more freedom of movement and positions possible.

The above list constitutes our observations of using wearable computers in telepresence. Many of the problems are commonly known in the field of wearable computing, yet their actual implications for telepresence have not been emphasized. Motivated by the need for the experience to be as effective and unbiased as possible, our conclusion is that the appearance and aesthetics of a wearable computer must be taken in consideration when planning to use such a platform for telepresence.

\subsection{Remote Interactions made Possible}

The remote interactions that the system allows is currently limited mainly to unidirectional communication, coming from the persons at the remote side to the local participants who receive it. The people at the remote location currently have no way of seeing the participants, as the remote user is "opaque" in that sense. Participants who wish to speak with remote persons must do so through the user of the wearable computer, who serves as a mediator for the communication. This is further described in [15] where we utilize this opacity in the Knowledgeable User concept, where the remote user effectively becomes a representative for the shared knowledge of the other participants. Except for the option of adding a speaker to the wearable computer, thus allowing participants to speak directly with remote persons, we do not have any plans to allow for bidirectional interaction. Rather, we remain focused on providing an ambient sense of presence to the remote user as well as the participants.

\subsection{Summary}

We will summarize this evaluation of our wearable telepresence system in three statements, serving as advice for those who wish to reproduce and deploy a similar system.

- The time to prepare, setup and use the system will influence how much it will be used in everyday situations, warranting the design of a streamlined system if an investment in such technology is to be made.

- A participant can easily shift between different levels of immersion, and even with relatively unsophisticated means get a good experience and interact with the remote environment.

- The aesthetical appearance of the wearable computing equipment should not be neglected, as this may otherwise influence the people at the remote location for better or for worse.

\section{CONCLUSIONS}

We have presented our experiences of using a wearable computer as a platform for telepresence, conveying the presence of a remote locations to the participants of a continuously running e-meeting session. Experiences in real-life scenarios such as fairs, events and everyday situations, have allowed us to identify shortcomings and subsequently address these to improve the platform. We have evaluated the platform in terms of overall usability, and motivated what is of importance for the audience's experience to be as seamless as possible. In the introduction, we posed three research questions which we will now summarize our answers to.
- The form of telepresence that can be provided using today's wearable computing technology can be very encompassing; even with an ordinary e-meeting application at the user's desktop, a fruitful experience can be delivered. For users who are already accustomed to enjoy the everyday presence of their fellow co-workers at their desktops, the step into mobile telepresence is a small one to take in order to extend its reach even further.

- To deliver a seamless experience of telepresence, the remote user must be able to freely interact with his environment, without social or technical obstacles that are not part of what should be conveyed. From a participant's point of view, having access to multiple interfaces (i.e. live, via the web, or via historical accounts) through which an event can be experienced, can be desirable in order for a seamless experience regardless of place and time.

- To simplify the deployment of wearable telepresence in everyday life, the remote user's equipment needs to be unobtrusive to handle and less noticeable, in order not to interfere with the remote environment. The user interface of the remote user must for this reason be highly efficient, while for participants an ordinary e-meeting application can serve to provide an experience that is good enough.

\subsection{Future Work}

We will redesign our current wearable computer prototype and fully incorporate the solutions suggested in this paper, in order to streamline the user's interaction with the wearable computer and the surrounding environment. The long term goal is to make remote interaction more efficient in general, allowing knowledge to pass back and forth between local and remote participants, either directly through the wearable technology itself or through the user of it acting as a mediator.

\section{ACKNOWLEDGMENTS}

This work was funded by the Centre for Distance-spanning Technology (CDT) under the VITAL Mål-1 project, and by the Centre for Distance-spanning Health care (CDH).

\section{REFERENCES}

[1] M. Billinghurst, J. Bowskill, M. Jessop, and J. Morphett. A wearable spatial conferencing space. In Proc. of the 2nd International Symposium on Wearable Computers, pages 76-83, 1998.

[2] M. Chen. Leveraging the asymmetric sensitivity of eye contact for videoconference. In Proceedings of the SIGCHI conference on Human factors in computing systems, pages 49-56. ACM Press, 2002.

[3] A. Clark. What do we want from a wearable user interface. In Proceedings of Workshop on Software Engineering for Wearable and Pervasive Computing, June 2000.

[4] N. Dahlbäck, A. Jönsson, and L. Ahrenberg. Wizard of oz studies: why and how. In Proceedings of the 1st international conference on Intelligent user interfaces, pages 193-200. ACM Press, 1993.

[5] S. R. Fussell, L. D. Setlock, and R. E. Kraut. Effects of head-mounted and scene-oriented video systems on remote collaboration on physical tasks. In Proceedings of the conference on Human factors in computing systems, pages 513-520. ACM Press, 2003. 
[6] S. K. Ganapathy, A. Morde, and A. Agudelo. Tele-collaboration in parallel worlds. In Proceedings of the 2003 ACM SIGMM workshop on Experiential telepresence, pages 67-69. ACM Press, 2003.

[7] K. Goldberg, D. Song, and A. Levandowski. Collaborative teleoperation using networked spatial dynamic voting. Proceedings of the IEEE, 91:430-439, March 2003.

[8] K. Goldberg, D. Song, Y. Khor, D. Pescovitz, A. Levandowski, J. Himmelstein, J. Shih, A. Ho, E. Paulos, and J. Donath. Collaborative online teleoperation with spatial dynamic voting and a human "tele-actor". In Proceedings of the IEEE International Conference on Robotics and Automation (ICRA'02), volume 2, pages 1179-1184, May 2002.

[9] N. P. Jouppi. First steps towards mutually-immersive mobile telepresence. In Proceedings of the 2002 ACM conference on Computer supported cooperative work, pages 354-363. ACM Press, 2002.

[10] K. Lyons and T. Starner. Mobile capture for wearable computer usability testing. In Proceedings of IEEE International Symposium on Wearable Computing (ISWC 2001), Zurich, Switerland, 2001.

[11] S. Mann. Wearable computing: A first step towards personal imaging. IEEE Computer, 30:25-32, February 1997.

[12] S. Mann. Personal imaging and lookpainting as tools for personal documentary and investigative photojournalism. ACM Mobile Networks and Applications, 4, March 1999.

[13] S. Mann and R. Picard. An historical account of the 'wearcomp' and 'wearcam' inventions developed for applications in 'personal imaging'. In IEEE Proceedings of the First International Conference on Wearable Computing, pages 66-73, October 1997.

[14] M. Nilsson, M. Drugge, and P. Parnes. In the borderland between wearable computers and pervasive computing. Research report, Luleå University of Technology, 2003. ISSN 1402-1528.
[15] M. Nilsson, M. Drugge, and P. Parnes. Sharing experience and knowledge with wearable computers. In Pervasive 2004: Workshop on Memory and Sharing of Experiences, April 2004.

[16] P. Parnes, K. Synnes, and D. Schefström. mStar: Enabling collaborative applications on the internet. Internet Computing, 4(5):32-39, 2000.

[17] R. Parviainen and P. Parnes. A Web Based History tool for Multicast e-Meeting Sessions. In Proceedings of the IEEE International Conference on Multimedia and Expo (ICME'2004), June 2004.

[18] R. Parviainen and P. Parnes. The MIM Web Gateway to IP Multicast E-Meetings. In Proceedings of the SPIE/ACM Multimedia Computing and Networking Conference (MMCN'04), 2004.

[19] E. Paulos. Connexus: a communal interface. In Proceedings of the 2003 conference on Designing for user experiences, pages 1-4. ACM Press, 2003.

[20] B. J. Rhodes. WIMP interface considered fatal. In IEEE VRAIS'98: Workshop on Interfaces for Wearable Computers, March 1998.

[21] T. Richardson, Q. Stafford-Fraser, K. R. Wood, and A. Hopper. Virtual network computing. IEEE Internet Computing, 2(1):33-38, 1998.

[22] N. Roussel. Experiences in the design of the well, a group communication device for teleconviviality. In Proceedings of the tenth ACM international conference on Multimedia, pages 146-152. ACM Press, 2002.

[23] J. Siegel, R. E. Kraut, B. E. John, and K. M. Carley. An empirical study of collaborative wearable computer systems. In Conference companion on Human factors in computing systems, pages 312-313. ACM Press, 1995.

[24] F. Tang, C. Aimone, J. Fung, A. Marjan, and S. Mann. Seeing eye to eye: a shared mediated reality using eyetap devices and the videoorbits gyroscopic head tracker. In Proceedings of the International Symposium on Mixed and Augmented Reality (ISMAR2002), pages 267-268, Darmstadt, Germany, Sep. 1 - Oct. 12002. 